asymptomatic individuals (all signposted via "Grindr"). Current work includes using "Grindr" to signpost users to our service, implementing online booking and expanding the use of POCT at community SHS. Clinics should consider using social media and geolocation-based apps in addition to traditional health promotion.

\section{P53 WITHDRAWN}

\section{P54 SYSTEMATIC REVIEW AND META-ANALYSIS OF RANDOMISED CONTROL TRIALS OF INTERACTIVE DIGITAL INTERVENTIONS FOR SEXUAL HEALTH PROMOTION}

${ }^{1}$ Sonali Wayal*, ${ }^{1}$ Julia Bailey, ${ }^{1}$ Elizabeth Murray, ${ }^{1}$ Greta Rait, ${ }^{1}$ Richard Morris, ${ }^{2}$ Richard Peacock, ${ }^{1}$ Irwin Nazareth. 'University College London, London, UK;

${ }^{2}$ The Whittington Hospital NHS Trust, London, UK

\section{$10.1136 /$ sextrans-2015-052126.97}

Background Digital technology offers potential for sexual health promotion.

Aims We conducted systematic review examining effectiveness of sexual health promotion interactive digital interventions (IDI) compared to 1) minimal interventions (e.g. leaflet); 2) face-toface interventions; 3) different IDI designs.

Methods IDI require users' contributions to produce personally relevant feedback. We searched 40 electronic databases for randomised controlled trials (RCT) of IDI for sexual health promotion from start dates to 30/04/2013. Separate meta-analyses were conducted for comparisons 1, 2, and 3, by outcome types (knowledge, self-efficacy, intention, sexual behaviour, biological outcomes) using random effects models. Subgroup analyses tested: age, risk grouping, setting (online, healthcare, educational).

Results We identified 36 RCTs (11,818 participants) from developed countries. Comparison 1: IDI improved knowledge ((standardised mean difference (SMD) 0.48 , 95\% CI 0.19 to 0.76 )); self-efficacy (SMD 0.11, 95\% CI 0.04 to 0.19), intention (SMD 0.13 , 95\% CI 0.05 to 0.22), sexual behaviour ((Odds Ratio (OR) $1.20,95 \%$ CI 1.02 to 1.41$)$ ), but not biological outcomes (OR $0.81,95 \%$ CI 0.56 to 1.16 ). IDI delivered in educational settings improved sexual behaviour (OR 2.09, 95\% CI 1.43 to 3.04), but not in healthcare settings (OR 1.17, 95\% CI 0.94 to 1.45 ), or online (OR $0.96,95 \%$ CI 0.79 to 1.17). Comparison 2: IDI improved knowledge (SMD 0.36, 95\% CI 0.13 to 0.58 ), intention (SMD $0.46,95 \%$ CI 0.06 to 0.85 ), but not self-efficacy (SMD 0.38, 95\% CI -0.01 to 0.77). Comparison 3: Tailoring had no effect on outcomes.
Conclusion IDIs can enhance knowledge, self-efficacy, intention, and sexual behaviour.

\section{P55 THE USE OF WEB-BASED TECHNOLOGY TO MEASURE PATIENT EXPERIENCE IN SEXUAL HEALTH SERVICES}

${ }^{1}$ Anna Hartley, ${ }^{1}$ Rebecca Marcus*, ${ }^{2}$ Shema Tariq, ${ }^{1}$ Johura Begum, ${ }^{1}$ Janice Purkis, ${ }^{1}$ Liat Sarner. ${ }^{1}$ Barts Health NHS Trust, London, UK; ${ }^{2}$ University College London, London, UK

\subsection{6/sextrans-2015-052126.98}

Introduction In comparison to other specialities, generating feedback from sexual health patients on clinic experience is challenging. Web-based technology can address many challenges associated with paper-based surveys, and is increasingly used to generate feedback in healthcare. A survey conducted in our service showed that four-fifths of our patients use smartphones; we therefore wanted to use technology to capture patient experience of our service.

Aim To measure real-time patient experience of our sexual health service using an online questionnaire.

Methods Since May 2014, new patients attending one of our five services are sent a link to an online survey via free text message. The short survey captures demographic data and feedback, with facility to request call back to discuss any concerns.

Results Since May 2014, 2457 new patients (18\%) have completed the survey $(2457 / 13753)$.

Discussion We have demonstrated high levels of satisfaction with our service as a result of this online survey. Implementation challenges include varying response rates, administration time and cessation of free messaging. However, the generation of real-time feedback is valued by staff, commissioners and patients, and has resulted in several service improvements e.g. improved signage and new processes for triaging patients.

\section{P56 ELECTRONIC PATIENT RECORDS (EPR) AND THE IMPACT ON ATTENDANCE WITHIN A LEVEL 3 SEXUAL HEALTH SERVICE}

Belinda Loftus*, Cheryl Robinson, Sophie Brady. Bradford Teaching Hospitals NHS Foundation Trust, Bradford, West Yorkshire, UK

\subsection{6/sextrans-2015-052126.99}

Background Staff complained that the introduction of the EPR in December 2013 slowed down their consultations and thought that attendances had reduced significantly as a result of having to "cap" walk in clinics and reduce the number of appointment slots. In the early months post implementation there were

\begin{tabular}{|c|c|c|c|c|c|}
\hline Clinic & Percentage responses & Male & Seen within 30 mins & Treated with dignity/respect (strongly agree/agree) & Would recommend service (strongly agree/agree) \\
\hline $\mathrm{C} 1$ & $(1090 / 7417) 15 \%$ & $48 \%$ & $49 \%$ & $97 \%$ & $94 \%$ \\
\hline $\mathrm{C} 2$ & $(493 / 2200) 22 \%$ & $35 \%$ & $24 \%$ & $93 \%$ & $90 \%$ \\
\hline C3 & (255/1605) $16 \%$ & $52 \%$ & $46 \%$ & $95 \%$ & $92 \%$ \\
\hline C4 & (276/1921) $14 \%$ & $38 \%$ & $22 \%$ & $96 \%$ & $88 \%$ \\
\hline C5 & (343/610 56\% & $15 \%$ & $50 \%$ & $90 \%$ & $81 \%$ \\
\hline
\end{tabular}

